\title{
Modeling of tip kinetics of undercooled Ti dendrites with consideration of forced flow and oxygen impurity effects
}

Dandan Zhao a , Jianrong $\mathrm{Gao}^{\mathrm{a},{ }^{*}}$, Andrew $\mathrm{Kao}^{\mathrm{b}}$

a Key Laboratory of Electromagnetic Processing of Materials (Ministry of Education), Northeastern University, Shenyang 110819, China

${ }^{\mathrm{b}}$ Centre for Numerical Modelling and Process Analysis, University of Greenwich, London SE10 9LS, United Kingdom

\begin{abstract}
Tip velocities of undercooled dendrites in electromagnetically levitated melt droplets of pure Ti were modeled using a theory on three-dimensional dendritic growth with fluid flow and a dilute solute. The modeling shows that a forced flow due to electromagnetic stirring depresses tip velocities of the dendrites at low undercoolings and that its effect becomes negligible at high undercoolings. In contrast, an oxygen impurity dissolved in liquid $\mathrm{Ti}$ depresses tip velocities of the dendrites over a broad range of undercooling while it coarsens tip radii. Such modeling results allowed for reconciliation of discrepancies in literature data by considering an oxygen impurity effect. The modeling also predicts that the effect of the oxygen impurity becomes insignificant when its concentration is reduced below $50 \mathrm{ppm}$ in atomic fraction.
\end{abstract}

Keywords: Dendrites; tip kinetics; modeling; liquid flow; oxygen impurity

* Corresponding author. E-mail: jgao@mail.neu.edu.cn (J. Gao). 


\section{Introduction}

Metal additive manufacturing technologies have received extensive attention because of advantages in producing geometrically complex or chemically graded parts over traditional technologies (see a recent review [1,2]). There is growing interest in an understanding of non-equilibrium solidification kinetics of a small melt pool for the purpose of online control of microstructure and defect formation and improvement of mechanical properties of as-built products $[3,4]$. In traditional non-equilibrium solidification processing, the cooling rate of a liquid is treated as the key process parameter. In additive manufacturing technologies, the scanning speed of a power laser or an electron beam is treated as a key process parameter instead. From a fundamental point of view, it is liquid undercooling that plays a critical role in determining metastable microstructure formation [5-8]. In situ observations using ultrafast X-ray imaging [9] demonstrated that a maximum solidification velocity of $0.75 \mathrm{~m} / \mathrm{s}$ was attained under a simulated condition of laser additive manufacturing technologies of $\mathrm{Ti}-6 \mathrm{Al}-4 \mathrm{~V}$ alloys. Such a high solidification velocity should be driven only by a high liquid undercooling. However, it is challenging to measure this quantity directly because of a high temperature gradient in the melt pool [10]. To bypass this difficulty, it is plausible to evaluate liquid undercooling by comparing an actual solidification velocity to an established crystal growth velocity-undercooling relationship. However, crystal growth velocities in an undercooled liquid are apt to fluid flow and impurities dissolved. The effects of fluid flow and impurities are not well determined for most of metals and alloys. Pure Ti is an example of this kind.

$\mathrm{Ti}$ is chemically reactive in its liquid state and a high undercooling of its bulk liquid can be accessed only by containerless melting and solidification [11-15]. Ti also has a high affinity to oxygen in solid and liquid states [16]. Thus, pure Ti commercially available typically has an oxygen impurity concentration of about $2500 \mathrm{ppm}$ in atomic fraction (1000 ppm in mass fraction) [17]. After special purification, high-purity Ti could have an oxygen concentration of $89 \mathrm{ppm}$ in atomic fraction (30 ppm in mass fraction) [18]. In solidification, the oxygen impurity dissolved in liquid Ti may slow down dendritic growth kinetics due to a solute drag effect. For this reason, measured tip velocities of undercooled Ti dendrites could be dependent on bulk concentration of an oxygen impurity. Walder and Ryder [11] found that tip velocities of Ti dendrites in electromagnetically levitated melt droplets were 
comparable to those of undercooled Ni dendrites under similar experimental conditions. Compared to pure $\mathrm{Ti}$, pure $\mathrm{Ni}$ can easily reach a low oxygen impurity concentration [8]. Thus, observations of similar tip velocities of the two metals suggested a low concentration of the oxygen impurity in their Ti samples. Bassler [12] measured tip velocities of Ti dendrites using a similar experimental set-up. Their data showed a depression of tip velocities as compared to those measured by Walder and Ryder [11]. It is unclear if the discrepancy is due to a difference in the oxygen impurity because impurity concentrations were not determined in either study. Very recently, Kavousi et al. modeled tip kinetics of undercooled $\mathrm{Ti}$ dendrites [19]. Their molecular dynamics simulation suggested an interfacial kinetic coefficient similar to that of pure $\mathrm{Ni}$, but their phase field modeling suggested tip velocities even lower than those measured by Bassler [12]. Thus, there remain discrepancies about the tip velocities of Ti dendrites in literature. Although a revisit to tip velocities in electromagnetically levitated melts may provide a straightforward approach to reconciling the discrepancies, it is easier and instructive to carry out modeling of observed tip velocities with consideration of effects of fluid flow and impurities $[8,20]$.

In this work, tip kinetics of undercooled $\mathrm{Ti}$ dendrites was modeled using a theory on three-dimensional dendritic growth [21] with consideration of a fluid flow effect and an oxygen impurity effect. The modeling suggested that a forced flow due to electromagnetic stirring has a significant effect on tip kinetics at low undercoolings but that this effect becomes negligible at high undercoolings. The modeling showed that the effect of a typical oxygen impurity of pure Ti on tip kinetics is significant at low and high undercoolings. Such modeling results reconciled the discrepancies about the tip velocities of Ti dendrites in electromagnetically levitated melts [11,12]. They also shed light onto effects of an oxygen impurity on microstructure formation in other materials. For convenience, we give a brief introduction to the theory first.

\section{Theory}

The interface undercooling at a primary tip of an undercooled dendrite can be divided into three parts [7]: a curvature undercooling $\Delta T_{r}$, a constitutional undercooling $\Delta T_{c}$, and a kinetic undercooling $\Delta T_{k}$. There is an additional undercooling, thermal undercooling $\Delta T_{t}$, in the liquid close to the tip. A sum of the interface and the thermal undercooling defines a 
bulk undercooling, $\Delta T$ that drives tip growth [7], namely

$$
\Delta T=\Delta T_{t}+\Delta T_{t}+\Delta T_{c}+\Delta T_{k} .
$$

Alexandrov and Galenko analyzed effects of fluid flow and a dilute solute on tip kinetics of an undercooled dendrite at an arbitrary Péclet number. Their theory, termed AG theory in short, considered three-dimensional dendritic growth with a laminar flow incident to a primary tip [21]. In the AG theory, the four partial undercoolings are expressed as

$$
\begin{aligned}
& \Delta T_{t}=\left(Q / C_{p}\right) I v\left(P_{t}, P_{f}\right), \\
& \Delta T_{r}=2 \Gamma / R, \\
& \Delta T_{c}=\frac{m_{e} C_{0}\left(1-k_{e}\right) P_{c} \exp \left(P_{c}+P_{c f}\right) I_{c}(\infty)}{1-\left(1-k_{e}\right) P_{c} \exp \left(P_{c}+P_{c f}\right) I_{c}(\infty)}, \\
& \Delta T_{k}=V / \mu,
\end{aligned}
$$

where $Q$ is the fusion of heat of the solid, $C_{p}$ is the specific heat of the liquid, $\Gamma$ is the Gibbs-Thompson coefficient of a solid-liquid interface, $R$ is the dendritic tip radius, $m_{e}$ is the liquidus slope, $C_{0}$ is the solute concentration (impurity concentration), $k_{e}$ is the equilibrium partition coefficient, $V$ is the dendritic tip velocity, and $\mu$ is the interfacial kinetic coefficient. $I v\left(P_{t}, P_{f}\right)$ and $I v\left(P_{c}, P_{c f}\right)$ are flow-dependent Ivantsov functions for heat transfer and mass transfer in the liquid, respectively and can be expressed as

$$
\begin{aligned}
& I v\left(P_{t}, P_{f}\right)=P_{t} \exp \left(P_{t}+P_{f}\right) I_{t}(\infty), \\
& I v\left(P_{c}, P_{c f}\right)=P_{c} \exp \left(P_{c}+P_{c f}\right) I_{c}(\infty),
\end{aligned}
$$

where $P_{t}$ is a thermal Pélect number, $P_{f}$ is a flow-dependent thermal Pélect number, $P_{c}$ is a chemical Péclet number, and $\mathrm{P}_{c f}$ is a flow-dependent chemical Péclet number. These Pélect numbers are defined as follows:

$$
\begin{aligned}
& P_{t}=V R /\left(2 D_{t}\right), \\
& P_{f}=U R /\left(2 D_{t}\right), \\
& P_{c}=V R /\left(2 D_{c}\right), \\
& P_{c f}=U R /\left(2 D_{c}\right),
\end{aligned}
$$

where $D_{t}$ is thermal diffusivity in the liquid, $D_{c}$ is chemical diffusivity in the liquid, and $U$ is flow velocity. The two integrals of Equations (6) and (7) can be expressed as

$$
I_{\mathrm{t}}(\eta)=\int_{1}^{\eta} \exp \left[2 P_{f} \int_{1}^{\eta^{\prime}} \frac{g\left(\eta^{\prime \prime}\right)}{\sqrt{\eta^{\prime \prime}}} d \eta^{\prime \prime}-\left(P_{f}+P_{t}\right) \eta^{\prime}\right] \frac{d \eta^{\prime}}{\eta^{\prime}},
$$




$$
I_{\mathrm{c}}(\eta)=\int_{1}^{\eta} \exp \left[2 P_{f} \frac{D_{t}}{D_{c}} \int_{1}^{\eta^{\prime}} \frac{g(\eta ")}{\sqrt{\eta^{\prime \prime}}} d \eta^{\prime \prime}-\left(P_{f}+P_{t}\right) \frac{D_{t}}{D_{c}} \eta^{\prime}\right] \frac{d \eta^{\prime}}{\eta^{\prime}},
$$

where the hydrodynamic function $g\left(\eta^{\prime \prime}\right)$ is given by

$$
g\left(\eta^{\prime \prime}\right)=\frac{\sqrt{\eta^{\prime \prime}} E_{1}\left(\operatorname{Re} \eta^{\prime \prime} / 2\right)}{2 E_{1}(\operatorname{Re} / 2)}+\frac{\exp (-\operatorname{Re} / 2)-\exp \left(-\operatorname{Re} \eta^{\prime \prime} / 2\right)}{\sqrt{\eta^{\prime \prime}} \operatorname{Re} E_{1}(\operatorname{Re} / 2)} .
$$

$R e$ is the Reynolds number and given by

$$
R e=R U \rho_{l} / v
$$

where $\rho_{l}$ and $v$ are mass density and dynamical viscosity, respectively.

The AG theory [21] includes a linear analysis of tip stability and determines the tip stability parameter as

$$
\sigma^{*}=\frac{\sigma_{0} \beta^{7 / 4}}{1+b\left(\alpha \beta^{-3 / 4}\right)^{11 / 4}}\left[\frac{1}{\left(1+a_{1} \sqrt{\beta} P_{t}\right)^{2}}+\frac{1}{\left(1+a_{2} \sqrt{\beta} P_{t} D_{t} / D_{c}\right)^{2}} \frac{2 m_{e} C_{i}\left(1-k_{e}\right) D_{t}}{\left(Q / C_{p}\right) D_{c}}\right]
$$

where $\sigma_{0}$ and $b$ are material-dependent stability parameters, $\beta=15 \varepsilon 4$ is the anisotropy of the liquid/solid interfacial energy with a four-fold symmetry $\varepsilon 4, a_{1} \approx 0.381 \sigma_{0}$ and $a_{2} \approx$ $0.505 \sigma_{0}$. The parameter $\alpha$ is given by

$$
\alpha=\frac{d_{0} U a(\mathrm{Re})\left[1+D_{c} /\left(2 D_{t}\right)\right]}{4 R V\left[D_{c} /\left(2 D_{t}\right)+m_{e} C_{i}\left(1-k_{e}\right) C_{p} / Q\right]},
$$

where $d_{0}$ is the thermocapillary length and $C_{i}$ is the solute concentration of the liquid at the dendritic tip. $a(R e)$ is a flow dependent function and given by

$$
a(\operatorname{Re})=\frac{\exp (-\operatorname{Re} / 2)}{E_{1}(\operatorname{Re} / 2)}
$$

where $E_{1}(q)=\int_{q}^{\infty} u^{-1} \exp (-u) d u$ is the first exponential integral function. The solute concentration $C_{i}$ is given by

$$
\Delta T_{c}=\frac{m_{e} C_{0}\left(1-k_{e}\right) P_{\mathrm{c}} \exp \left(P_{c}+P_{c f}\right) I_{c}(\infty)}{1-\left(1-k_{e}\right) P_{c} \exp \left(P_{c}+P_{c f}\right) I_{c}(\infty)} .
$$

Gao et al. [22] showed that the AG theory can provide a reasonable description of tip kinetics of undercooled succinonitrile dendrites under the influence of natural convection on the ground and in space. They further showed that the AG theory can also provide a reasonable description of tip kinetics of undercooled dendrites of pure Fe and pure Ni under 
the influence of strong magnetohydrodynamic flows [20,23]. Following such studies, it was supposed that a modeling of tip kinetics of undercooled Ti dendrites using the AG theory may help understand the discrepancies in literature $[11,12]$. Modeling results are shown below.

\section{Modeling of tip velocities of undercooled Ti dendrites}

Table 1 Parameters used in modeling of tip kinetics of thermal dendrites of Ti.

\begin{tabular}{|c|c|c|c|}
\hline Parameter & Symbol & Value & References \\
\hline Heat of fusion $\left(\mathrm{J} \mathrm{mol}^{-1}\right)$ & $Q$ & 14300 & [13] \\
\hline Liquid specific heat $\left(\mathrm{J} \mathrm{mol}^{-1}\right)$ & $C_{p}$ & 45.5 & {$[13]$} \\
\hline Melting temperature (K) & $T_{m}$ & 1943 & {$[13]$} \\
\hline Thermal diffusivity $\left(\mathrm{m}^{2} \mathrm{~s}^{-1}\right)$ & $D_{t}$ & $9.5 \times 10^{-6}$ & {$[15]$} \\
\hline Density of liquid $\left(\mathrm{kg} \mathrm{m}^{-3}\right)$ & $\rho_{l}$ & 4130 & [15] \\
\hline Kinetic viscosity in liquid (Pa s) & $v$ & $3.3 \times 10^{-3}$ & {$[15]$} \\
\hline Gibbs-Thompson coefficient (K m) & $\Gamma$ & $2.77 \times 10^{-7}$ & * \\
\hline Interfacial kinetic coefficient $\left(\mathrm{m} \mathrm{s}^{-1} \mathrm{~K}^{-1}\right)$ & $\mu$ & 0.37 & present work \\
\hline Anisotropy of surface tension & $\varepsilon_{4}$ & 0.04 & [19] \\
\hline Tip stability parameter & $\sigma_{0}$ & 0.04 & present work \\
\hline Tip stability parameter & $b$ & 0.1 & {$[20-23]$} \\
\hline Flow velocity $\left(\mathrm{m} \mathrm{s}^{-1}\right)$ & $U$ & -0.3 & [20] \\
\hline
\end{tabular}

* Calculated in terms of a solid/liquid interfacial energy of $\sigma_{s / l}=0.176 \mathrm{~J} \mathrm{~m}^{-2}[19]$.

Diffusion-controlled growth of thermal dendrites of pure Ti in stagnant flow conditions was modeled using the parameters listed in Table 1 and compared to the experimental data by Walder and Ryder [11]. For thermal dendrites, the constitutional undercooling $\Delta T_{c}=0$. Because forced flows have a negligible effect on tip velocities at high $\Delta T$ [20], least-square fitting of the AG theory to the experimental data in this undercooling region allowed for unambiguous determination of interfacial kinetic coefficient and tip stability parameter. In fitting, the relative error of an observed tip velocity was considered, which was defined as 
its percentage deviation from a modeled tip velocity at a given $\Delta T$. A sum of the square of the relative error of the experimental data over high $\Delta T$ was achieved by choosing an interfacial kinetic coefficient of $\mu=0.37 \mathrm{~m} / \mathrm{s} / \mathrm{K}$ and a tip stability parameter of $\sigma_{0}=0.04$. Provided that these two values are accepted, the AG theory gives a reasonably good prediction of the tip velocities observed at $\Delta T>50 \mathrm{~K}$. That is, the experimental data had a r.m.s. deviation of up to $20 \%$ only. Although $\mu$ is only a half of the value predicted by molecular dynamics simulation [19], it is comparable to a value of $0.30 \mathrm{~m} / \mathrm{s} / \mathrm{K}$ predicted and verified for pure Fe $[23,24]$. This similar magnitude is reasonable because Fe and Ti have similar melting temperatures and both crystallize into a body-centered cubic structure. The molecular dynamics simulation [19] may have overestimated $\mu$. On the other hand, $\sigma_{0}$ determined is by a factor of four larger than that of Fe. This difference is understood in terms of a larger anisotropy of liquid/crystal interfacial energy [19] and higher tip velocities of Ti dendrites. There is a discrepancy between the modeling and the experiment in a low undercooling region. The r.m.s. deviation of the experimental data amounted to $110 \%$ at $\Delta T<50 \mathrm{~K}$. Although solidification paths of the sample were not controlled by triggering of crystal nucleation, this discrepancy cannot be solely attributed to experimental errors. The thermal conductivity used in the modeling has high accuracy because it was measured by containerless processing of liquid and solid Ti in an ultrahigh vacuum [15]. Thus, it is neither the reason for the discrepancy. A forced flow effect due to electromagnetic stirring was determined to be significant on tip velocities of Ni dendrites at low $\Delta T$ [20]. Thus, it may be the reason for the discrepancy. To check this hypothesis, we considered the effect of a similar flow velocity of $U=-0.3 \mathrm{~m} / \mathrm{s}$ [20]. To check this hypothesis, we considered the effect of a similar flow velocity of $U=-0.3 \mathrm{~m} / \mathrm{s}[20]$. The flow was supposed to point to the tip growth direction and has the negative sign. According to Hyers [25], forced flows in an electromagnetically levitated Ni melt have a Re larger than 600 in the ground-based gravity condition and have a turbulent nature. Such a large $R e$ is defined on a length scale equal to the size of the levitated melt and provides a reason for a maximum flow velocity of up to $0.3 \mathrm{~m} / \mathrm{s}$. In the AG theory, Re is defined on a length scale equal to primary tip radius of an equiaxed dendrite (see Eq. 15) and has a much smaller value, e.g. $R e=0.68$ at $\Delta T \sim 26 \mathrm{~K}$. Thus, the forced flow was treated as a laminar flow in the modeling. Including this convective transport effect did not further reduce the deviations of the experimental 
data at higher $\Delta T$. Rather, it reduced the r.m.s. deviation of the experimental data to $46 \%$ at $\Delta T<50 \mathrm{~K}$. In other words, it brought about an improved agreement between the experiment and the modeling. A remaining difference can be attributed to reasonable experimental errors estimated from the scatter of the observed tip velocities at low $\Delta T$.

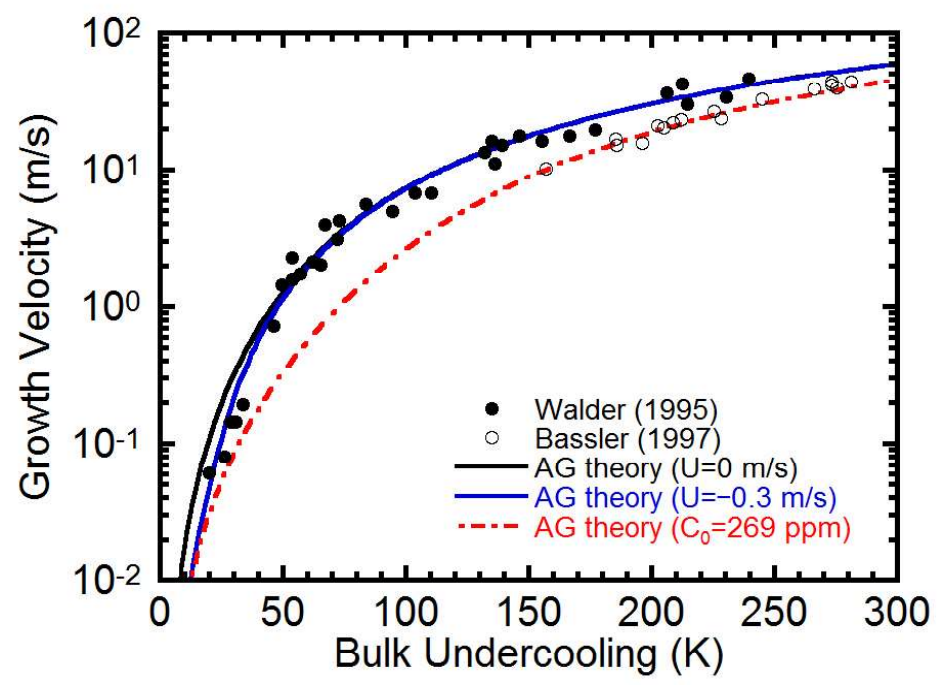

Fig. 1. Modeling of tip velocities of undercooled dendrites in electromagnetically levitated pure Ti. Solid and open circles are literature data $[11,12]$.

However, even when the flow effect is included, there is a discrepancy between the modeling of thermal dendrites and the tip velocities observed by Bassler [12]. There is no physical reason for consideration of a flow velocity higher than $0.3 \mathrm{~m} / \mathrm{s}$ to reconcile this discrepancy. Rather, it is more reasonable to consider an impurity effect. Although the species of impurities of the Ti material investigated by Bassler was not known, the majority impurity was most likely to be oxygen following other studies of pure Ti $[17,18]$. When oxygen is dissolved in levitated melts as the majority impurity, undercooled Ti dendrites crystallized therein should be treated as chemical dendrites. In other words, the $\Delta T_{c}$ of the dendritic tip is not zero and needs to be accounted for. Using parameters listed in Table 2, we modeled the tip velocities of chemical dendrites of Ti. Because a smaller atom usually diffuses faster than a large one, we adopted a value of $D_{c}=1 \times 10^{-8} \mathrm{~m}^{2} / \mathrm{s}$ for chemical diffusivity of oxygen in liquid $\mathrm{Ti}$. This value is higher than that of typical solute in a liquid metal [26]. As a first approximation, thermophysical properties of dilute Ti-O alloys 
including $\mu$ were supposed to be equal to those of oxygen-free Ti. The only unknown parameters then are $C_{0}$ and $\sigma_{0}$. Treating these parameters as free parameters, least-square fitting of the AG theory to the tip velocities observed by Bassler [12] yielded $\sigma_{0}=0.0078$ and $C_{0}=269 \mathrm{ppm}$ (atomic fraction). As these values are accepted, a r.m.s. deviation of the experimental data relative to the modeling is reduced down to $8 \%$. Such a small deviation indicates a fairly good agreement between the AG theory and the experiment by Bassler [12]. Note that the determined $\sigma_{0}$ value is by a factor of 5 smaller than that used in the modeling of the data measured by Walder and Ryder [11]. Such a large difference is due to the depression of tip velocities by the oxygen impurity (see Fig. 1). On the other hand, the determined $C_{0}$ is close to a reported value of $95 \mathrm{ppm}$ (mass fraction) for high-purity $\mathrm{Ti}$ [27]. For such reasons, the values of the two fitting parameters are reasonable.

Table 2 Parameters used in modeling of tip kinetics of chemical dendrites of Ti.

\begin{tabular}{llll}
\hline Parameter & Symbol & Value & References \\
\hline Heat of fusion $\left(\mathrm{J} \mathrm{mol}^{-1}\right)$ & $Q$ & 14300 & {$[13]$} \\
Liquid specific heat $\left(\mathrm{J} \mathrm{mol}^{-1}\right)$ & $C_{p}$ & 45.5 & {$[13]$} \\
Melting temperature $(\mathrm{K})$ & $T_{m}$ & 1943 & {$[13]$} \\
Thermal diffusivity $\left(\mathrm{m}^{2} \mathrm{~s}^{-1}\right)$ & $D_{t}$ & $9.5 \times 10^{-6}$ & {$[15]$} \\
Density of liquid $\left(\mathrm{kg} \mathrm{m}^{-3}\right)$ & $\rho_{l}$ & 4130 & {$[15]$} \\
Kinetic viscosity in liquid $(\mathrm{Pa} \mathrm{s})$ & $v$ & $3.3 \times 10^{-3}$ & {$[15]$} \\
Gibbs-Thompson coefficient $(\mathrm{K} \mathrm{m})$ & $\Gamma$ & $2.77 \times 10^{-7}$ & $*$ \\
Interfacial kinetic coefficient $\left(\mathrm{m} \mathrm{s}^{-1} \mathrm{~K}^{-1}\right)$ & $\mu$ & 0.37 & present work \\
Anisotropy of surface tension & $\varepsilon_{4}$ & 0.04 & {$[19]$} \\
Tip stability parameter & $\sigma_{0}$ & 0.0078 & present work \\
Tip stability parameter & $b$ & 0.1 & {$[20-23]$} \\
Chemical diffusivity $\left(\mathrm{m}^{2} \mathrm{~s}^{-1}\right)$ & $D_{c}$ & $1 \times 10^{-8}$ & present work \\
Oxygen concentration $($ at.\%) & $C_{0}$ & 0.0269 & present work \\
Equilibrium partition coefficient & $k_{e}$ & 1.6 & {$[16]$} \\
Liquidus slope $(\mathrm{K} /$ at.\%) & $\mathrm{m}_{\mathrm{e}}$ & 10 & {$[16]$} \\
\hline
\end{tabular}

* Calculated in terms of a solid/liquid interfacial energy of $\sigma_{\mathrm{S} / l}=0.176 \mathrm{~J} \mathrm{~m}^{-2}[19]$. 


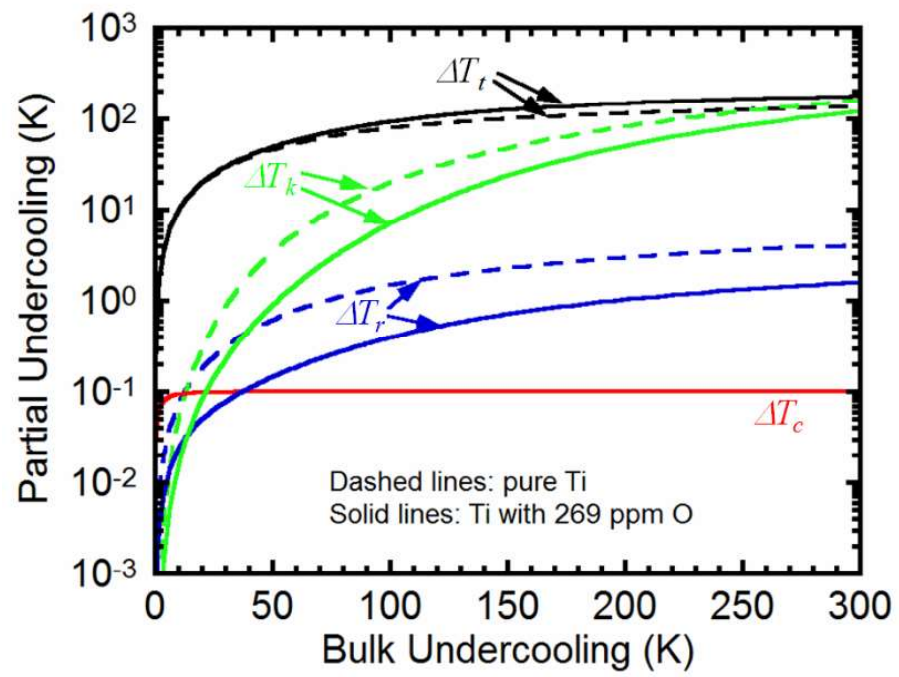

Fig. 2. Comparison of partial undercoolings of thermal dendrites and chemical dendrites of Ti.

Given such a good agreement between the Bassler's data [12] and the AG theory, the modeling provided insights into the effect of the oxygen impurity on the tip velocities of undercooled $\mathrm{Ti}$ dendrites. Like that modeled for thermal dendrites, the flow effect is significant for chemical dendrites at low $\Delta T$ (not shown in Fig. 1) and becomes insignificant at high $\Delta T$. Thus, attention was focused onto the impurity effect. Fig. 2 shows a comparison of partial undercoolings of the thermal and chemical dendrites of Ti. The free growth of the thermal dendrites of $\mathrm{Ti}$ is controlled largely by heat diffusion in the undercooled liquid. While this is true up to a maximum undercooling of $250 \mathrm{~K}$ accessed in the experiment [11], interface kinetics becomes important at $\Delta T>150 \mathrm{~K}$. The curvature undercooling $\Delta T_{r}$ plays a sub-critical role at $\Delta T<20 \mathrm{~K}$ but it becomes smaller than $\Delta T_{k}$ at higher $\Delta T$. The dissolution of the oxygen impurity in the liquid brings about significant changes of tip kinetics and partial undercoolings. As suggested above, the oxygen impurity introduces a non-zero $\Delta T_{c}$ to the $\Delta T$. The $\Delta T_{c}$ is saturated at $\Delta T \sim 10 \mathrm{~K}$ and its contribution is significant only at $\Delta T<50 \mathrm{~K}$. Because of this non-zero $\Delta T_{c}$, tip velocities are depressed at low $\Delta T$. However, the low undercooling region is also where the flow effect is significant. The depression of tip velocities induced by the dissolved oxygen impurity is comparable to that by the forced flow in the tip growth direction. Because of this similarity, it becomes 
technically difficult to distinguish the two effects in the low undercooling region. The two effects have to be distinguished by extending a modeling of tip velocities observed in the high undercooling region to the low undercooling region. The dissolved oxygen impurity has opposite effects on other partial undercoolings. Unlike a reduction of $\Delta T_{r}$ and $\Delta T_{k}, \Delta T_{t}$ gains an increase. Such opposing effects on partial undercoolings remain significant as $\Delta T$ increases. This is the very reason why the data measured by Bassler [12] became lower than those measured by Walder and Ryder [11].

\section{Discussion}

The above modeling revealed a significant effect of the oxygen impurity on tip kinetics of Ti dendrites at low and high $\Delta T$. There is a question at which concentration level the effects of oxygen impurity on the tip kinetics of Ti dendrites become insignificant. To answer this question, we extended the modeling of chemical dendrites to lower oxygen concentrations. For simplicity, we neglected the oxygen concentration of Walder and Ryder's samples and considered a power law dependence of $\sigma_{0}$ on $C_{0}$ :

$$
\sigma_{0}=0.04 \exp \left(-60.86 C_{0}\right) \text {, }
$$

where $C_{0}=0-269$ ppm. Using Eq. 20, tip velocities and tip radii of chemical dendrites of Ti at varied $C_{0}$ were calculated in a diffusive growth condition. As shown in Fig. 3, tip velocities of chemical dendrites are reduced generally as $C_{0}$ is increased. Meanwhile the tip radii of chemical dendrites are increased. When $C_{0}$ is increased from 0 to $50 \mathrm{ppm}$ (atomic fraction), the tip velocity at $\Delta T=26 \mathrm{~K}$ is depressed by about $25 \%$. This small depression is not significant compared to uncertainties of the data measured by Walder and Ryder [11]. Thus, an oxygen concentration of $C_{0}=50 \mathrm{ppm}$ can be accepted as a technical threshold for distinguishing the oxygen impurity effect. Strictly speaking, the oxygen impurity concentration of the samples investigated by Walder and Ryder was unlikely to be zero. Our modeling with a reasonably low oxygen impurity concentration, e.g. $C_{0}=100$ ppm in atomic fraction, still supports the conclusion of the forced flow effect and allows for the reconciliation of the discrepancies in literature [11,12].

The above modeling also provided insights into oxygen impurity effects observed in other metals. Battersby et al. [28] showed that an oxygen concentration of $2400 \mathrm{ppm}(600$ ppm in mass fraction) brought about a more than $50 \%$ reduction of tip velocities of $\mathrm{Cu}$ 
(a)

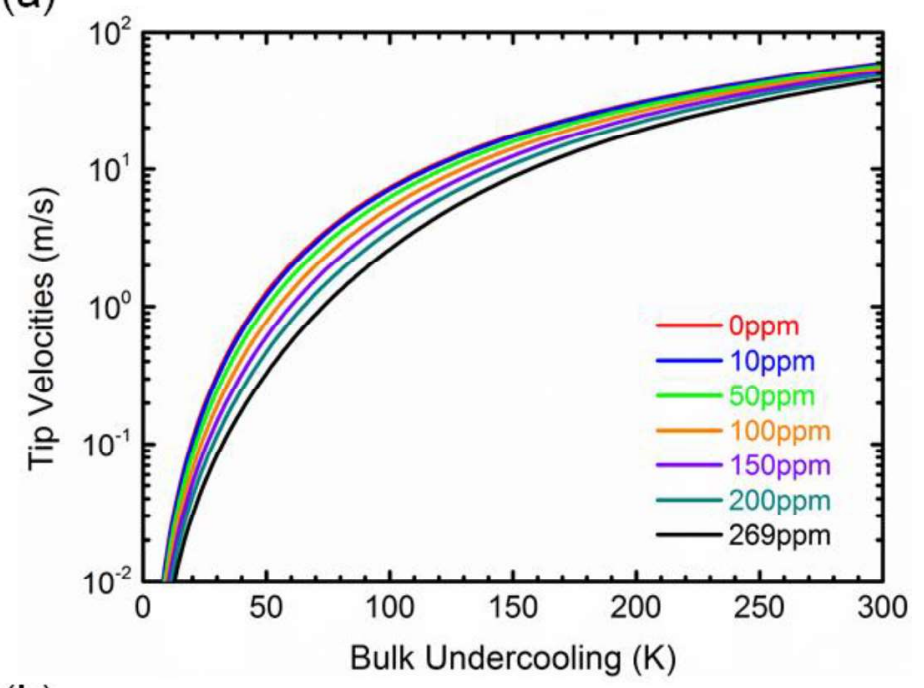

(b)

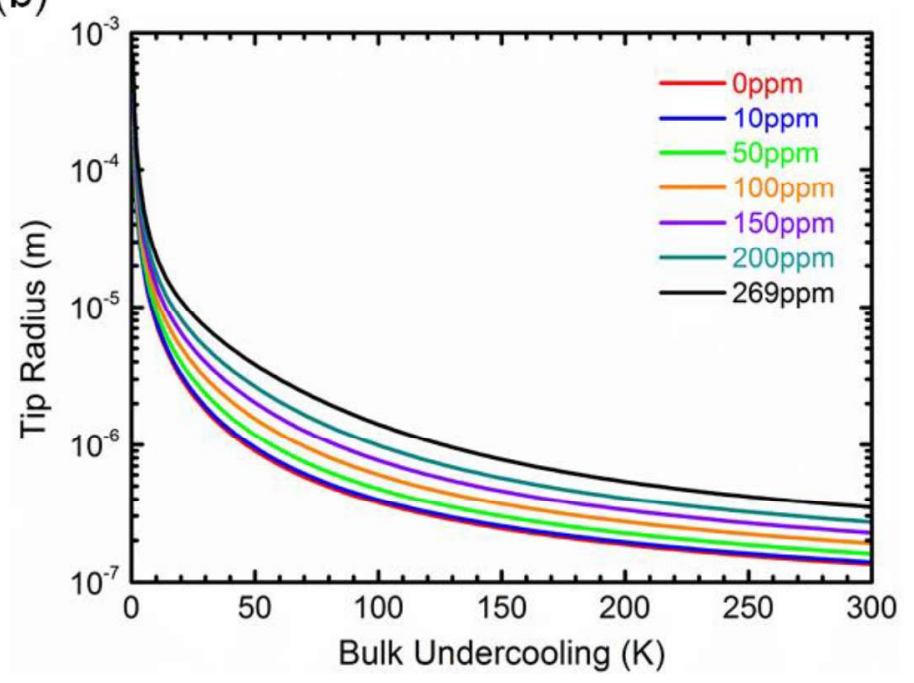

Figure 3. Modeled tip velocities (a) and tip radii (b) of Ti dendrites for different $\mathrm{O}$ concentrations. No flow effects are considered in the modeling for simplicity.

dendrites in a glass-fluxed melt, where convective flow is weaker than in a levitated melt [20]. Such a reduction of the tip velocities by the oxygen impurity can be understood in terms of the modeling results shown in Fig. 3a. In addition to tip velocities, the oxygen impurity may have a significant effect on tip radii of chemical dendrites and therefore on microstructure formation in non-equilibrium solidification. As shown in Fig. 3b, the change of the tip radii at medium $\Delta T$ due to an oxygen impurity is most significant. The introduction of an oxygen concentration of $C_{0}=269 \mathrm{ppm}$ as modeled above is shown to 
increase the tip radii by one order of magnitude at medium $\Delta T$. According to a model for grain-refinement in undercooled solidification [29], this huge change of the dendritic tip radii may increase a critical time required for break-up of a primary dendrite in a mushy zone and therefore shifts the formation of a grain-refined microstructure to a higher $\Delta T$ or a higher tip velocity. Consideration of this oxygen impurity effect can explain why the literature data of a critical $\Delta T$ for formation of a grain-refined microstructure in pure $\mathrm{Cu}$ and pure Ni showed a scatter [30,31].

As far as dendritic growth in an additive manufactured material of pure Ti is concerned, an oxygen impurity may have multiple effects on microstructure formation. Firstly, a depression of the tip velocity by the oxygen impurity may increase the time available for another dendrite to be nucleated from the liquid such that microstructure formation becomes more complex due to competition between neighboring dendrites [32]. Secondly, the depression of the tip velocity may also increase the sensitivity of dendritic growth to a liquid flow affecting local microstructure and defect formation. Lastly, coarsening of dendritic tip radii due to the impurity effect may increase secondary arm spacings because of a change of local thermal and chemical gradients. All of these effects on local or general microstructure formation may have an impact on mechanical properties of as-built material of pure Ti and thus, need to be investigated in the future.

\section{Conclusions}

Tip velocities of undercooled dendrites in electromagnetically levitated Ti melts have been modeled using a three-dimensional theory of dendritic growth with consideration of effects of a forced flow in the tip growth direction and an oxygen impurity. The modeling has shown that a typical oxygen impurity concentration in nominally high-purity $\mathrm{Ti}$ material causes a depression of tip velocities and coarsening of tip radii. Consideration of this oxygen impurity effect has reconciled the discrepancies in literature $[11,12]$. The modeling has also shown that the oxygen impurity effect on tip velocities of Ti dendrites is similar to that of a forced flow due to electromagnetic stirring at low undercoolings and that distinguishing of them requires an extension of the modeling of tip velocities at high undercoolings down to low undercoolings. The modeling has predicted that the effect on tip velocities becomes indiscernible when the oxygen impurity concentration of pure $\mathrm{Ti}$ is 
reduced below $50 \mathrm{ppm}$ (atomic fraction). Therefore, the oxygen impurity could have a significant influence in additive manufacturing technologies, the consequences of which need to be addressed in the future.

\section{Acknowledgement}

This work is financially supported by the National Natural Science Foundation of China (grant number [51271055]).

\section{References}

[1] C. Körner, Additive manufacturing of metallic components by selective electron beam melting — a review, Int. Mater. Rev. 61 (2016) 361-377.

[2] D. Herzog, V. Seyda, E. Wycisk, C. Emmelmann, Additive manufacturing of metals, Acta Mater. 117 (2016) 371-392.

[3] D.D. Gu, Y.C. Hagedorn, W. Meiners, G.B. Meng, R.J.S. Batista, K. Wissenbach, R. Poprawe, Densification behavior, microstructure evolution, and wear performance of selective laser melting processed commercially pure titanium, Acta Mater. 60 (2012) 3849-3860.

[4] H. Attar, M. Calin, L.C. Zhang, S. Scudion, J. Eckert, Manufacture by selective laser melting and mechanical behavior of commercially pure titanium, Mater. Sci. Eng. A 593 (2014) 170-177.

[5] K. Eckler, R.F. Cochrane, D.M. Herlarch, B. Feuerbacher, Evidence for a transition from diffusion-controlled to thermally controlled solidification in metallic alloys, Phys. Rev. B 45 (1992) 5019-5022.

[6] A.F. Norman, K. Eckler, A. Zambon, F. Gätner, S.A. Moir, E. Ramous, D.M. Herlach, A.L. Greer, Application of microstructure-selection maps to droplet solidification: a case study of the Ni-Cu system, Acta Mater. 46 (1998) 3355-3370.

[7] D.M. Herlach, Non-equilibrium solidification of undercooled metallic melts, Metals 4 (2014) 196-234.

[8] O. Funke, G. Phanikumar, P.K. Galenko, L. Chernova, S. Reutzel, M. Kolbe, D.M. Herlach, Dendrite growth velocity in levitated undercooled nickel melts, J. Cryst. Growth 297 (2006) 211-222. 
[9] C. Zhao, K. Fezzaa, R.W. Cunningham, H. Wen, F. De Carlo, L. Chen, A.D. Rollett, T. Sun, Real-time monitoring of laser powder bed fusion process using high-speed Xray imaging and diffraction, Sci. Rep. 7 (2017) 3602.

[10] U.S. Bertoli, G. Guss, S. Wu, M.J. Matthews, J.M. Schoenung, In-situ characterization of laser-powder interaction and cooling rates through high-speed imaging of powder bed fusion additive manufacturing, Mater. Design 135 (2017) 385-396.

[11] S. Walder, P.L. Ryder, A simple technique for the measurement of dendritic growth rates in undercooled metallic melts and its application to Ni and Ti, Mater. Sci. Eng. A 203 (1995) 197-202.

[12] B.T. Bassler, The solidification velocity of underooled metals and alloys, PhD thesis, Vanderbilt University, 1997.

[13] P.-F. Paradis, W.-K. Rhim, Non-contact measurements of thermophysical properties of titanium at high temperature, J. Chem. Thermodynamics 32 (2000) 123-133.

[14] G.W. Lee, S. Jeon, C. Park, D.-H. Kang, Crystal-liquid interfacial free energy and thermophysical properties of liquid Ti using electrostatic levitation: hypercooling limit, specific heat, total hemispherical emissivity, density and interfacial free energy, J. Chem. Thermodynmics 63 (2013) 1-6.

[15] K. Zhou, H.P. Wang, J. Chang, B. Wei, Expeirmental study of surface tension, specific heat and thermal diffusivity of liquid and solid titanium, Chem. Phys. Lett. 639 (2015) $105-108$.

[16] J.J. Muarray, H.A. Wriedt, The O-Ti (oxygen-titanium) system, Bull. Alloy Phase Diagrams 8 (1987) 148-165.

[17] D.H. Shin, I. Kim, J. Kim, Y.S. Kim, S.L. Semiatin, Microstructure development during equal-channel angular pressing of titanium, Acta Mater. 51 (2003) 983-996.

[18] C. Ouchi, H. Iizumi, S. Mitao, Effects of ultra-high purification and addition of interstitial elements on properties of pure titanium and titanium alloy, Mater. Sci. Eng. A 243 (1998) 186-195.

[19] S. Kavousi, B.R. Novak, M.A. Zaeem, D. Moldovan, Combined molecular dynamics and phase field simulation investigations of crystal-melt interfacial properties and dendritic solidification of highly undercooled titanium, Comput. Mater. Sci. 163 (2019) 218-229. 
[20] J. Gao, A. Kao, V. Bojarevics, K. Pericleous. P.K. Galenko, D.V. Alexandrov, Modeling of convection, temperature distribution and dendritic growth in glass-fluxed nickel melts, J. Cryst. Growth 471 (2017) 66-72.

[21] D.V. Alexandrov, P. Galenko, Dendrite growth under forced convection: analysis methods and experimental test, Uspekhi 57 (2014) 771-786.

[22] J. Gao, M.K. Han, A. Kao, K. Pericleous, D.V. Alexandrov, P.K. Galenko, Dendritic growth velocities in an undercooled melt of pure nickel under static magnetic fields: a test of theory with convection, Acta Mater. 103 (2016) 184-191.

[23] R.J. Zhao, J. Gao, A. Kao, K. Pericleous, Measurements and modelling of dendritic growth velocities of pure $\mathrm{Fe}$ with thermoelectric magnetohydrodynamics convection, J. Cryst. Growth 475 (2017) 354-361.

[24] D.Y. Sun, M. Asta, J.J. Hoyt. Crystal-melt interfacial free energies and mobilities in fcc and bcc Fe, Phys. Rev. B 69 (2004) 174103.

[25] R.W. Hyers, Fluid flow effects in levitated droplets, Meas. Sci. Technol. 16 (2005) $391-401$.

[26] Y. Liu, Z.Y. Long, H.B. Wang, Y. Du. B.Y. Huang, A predictive equation for solute diffusivity in liquid metals, Scripta Mater. 55 (2006) 367-370.

[27] A.A. Salem, S.R. Kalidindi, R.D. Doherty, Strain hardening regimes and microstructure formation during large strain compression of high purity titanium, Scrip. Mater. 46 (2002) 419-423.

[28] S.E. Battersby, R.F. Cochrane, A.M. Mullis, Microstructural evolution and growth velocity-undercooling relationships in the systems $\mathrm{Cu}, \mathrm{Cu}-\mathrm{O}$ and $\mathrm{Cu}-\mathrm{Sn}$ at high undercooling, J. Mater. Sci. 35 (2000) 1365-1373.

[29] M. Schwarz, A. Karma, K. Eckler, D.M. Herlach, Physical mechanism of grain refinement in solidification of undercooled melts, Phys. Rev. Lett. 73 (1994) $1380-1383$.

[30] B.T. Bassler, W.H. Hofmeister, G. Carro, B.J. Bayuzick, The velocity of solidification of highly undercooled nickel, Metall. Mater. Trans. A 25 (1994) 1301-1308.

[31] D. Li, K. Eckler, D.M. Herlach, Development of grain structures in highly undercooled germanium and copper, J. Cryst. Growth 160 (1996) 59-65. 
[32] Y. Wang, J. Shi, Y. Liu, competitive grain growth and dendrite morphology evolution in selective laser melting of Inconel 718 superalloy, J. Cryst. Growth 521 (2019) 1529. 